\title{
THE PARTICIPATION OF GIRLS AND BOYS FROM AGES 10 TO 18 IN STRUCTURED SPORTS AND EXTRA-CURRICULAR ACTIVITIES IN THE ASPECT OF SOCIAL AND ECONOMIC CONDITIONS
}

\author{
AGNIESZKA DMITRUK ${ }^{1}$, HELENA POPŁAWSKA ${ }^{1}$, KRYSTYNA GÓRNIAK ${ }^{2}$, WOJCIECH HOŁUB ${ }^{3}$ \\ Józef Piłsudski University of Physical Education in Warsaw, \\ Faculty of Physical Education and Sport in Biała Podlaska, Department of Biology and Anatomy', \\ Department of Correction and Compensation, \\ Faculty of Tourism and Health in Biała Podlaska, Department of Cosmetology ${ }^{3}$
}

\begin{abstract}
Mailing address: Agnieszka Dmitruk, Faculty of Physical Education and Sport, Department of Biology and Anatomy, 2 Akademicka Street, 21-500 Biała Podlaska, tel.: +48 83 3428738, fax: +48 83 3428800, e-mail: agnieszka.dmitruk@awf-bp.edu.pl
\end{abstract}

\begin{abstract}
Introduction. The importance of socio-economic factors in differentiating the physical activities of children and teenagers keeps changing. That is why the goal of this research was to identify certain social variables amongst those listed most often which differentiate the level of targeted physical activity of urban children and youths from the Bialskie district. Material and methods. The research was conducted on 1.084 students between the ages of 10 and 18 . Three age groups were chosen: 10 to 12, 13 to 15, and 16 to 18. Information about the attendance of respondents in Physical Education classes and structured extra-curricular sport activities, as well as preferred forms of physical activities were collected via a diagnostic survey. The same method was used in the assessment of the socio-economic status of the families of the respondents. The relations between attendance in extra-curricular sports activities and socio-economic factors was assessed by a multifactoral logistic regression model, and the statistical relevance of the differences was calculated by the Wald test. Results. Attendance in structured extra-curricular physical activity depended mostly on income per family member. In the case of type of work performed by parents, the attendance rate in both boys and girls was higher if the parents performed intellectual work. Parents' education and number of children in the family did not have a high influence on the attendance of respondents in structured extra-curricular sport activities. Conclusions. Increasing disproportions in societal prosperity can be an important factor limiting the attendance of the poorest group of children and youths in structured physical activities. The lack of influence on physical activity of such socio-economic variables as parents' education and the number of children in the family can be connected with the change of meaning of those factors in modern society (depreciation of the importance of higher education, decreased fertility).
\end{abstract}

Key words: physical activity, socio-economic factors, children and youths

\section{Introduction}

Physical activity plays a fundamental role in providing one with psychophysical well-being. It favorably affects mineralization and growth of bones as well as the functioning of the circulatory system, respiratory system, nervous system and endocrine system; it contributes to the increase of immunity of the organism [1, 2, 3, 4]. It also favorably effects the social and emotional development of children and youths [5, 6].

The World Health Organization recommends adults undertake moderate physical activity at least for 150 minutes a week, or intense physical activity for at least 75 minutes. Youths on the other hand should exercise at least 60 minutes a day with moderate or high intensity [7]. Nowadays the level of physical activity in many European countries is extremely insufficient for ensuring optimal health. Only 9\% of adult residents of the European Union undertake sport or exercise at least 5 times a week and $27 \%$ undertake other forms of physical activity. In Poland, $6 \%$ of the population play sports and only $26 \%$ regularly undertake other forms of physical activity. What is also disturbing is the fact that $49 \%$ of Polish people do not do any sport and $17 \%$ do not undertake any form of physical recreation [8]. Polish youths also do not reach the recommended level of physical activity. Data collected by Health Behavior in School-aged Children [9] show that between 2009 and 2010 an adequate level of physical activity was declared by $31 \%$ of boys aged $11,22 \%$ of boys aged 13 and 23\% aged 15 . The percentage of regularly exercising girls was smaller and decreased in the following researched age groups (from $23 \%$ at 11 to $10 \%$ at 15 ). Those values are close to the European average.

An indisputable role in undertaking physical activity by children is played by their parents and their attitude towards physical exercise as well as their school and peers. Many papers proved that parents' physical activity influences the activity of their children $[10,11,12]$. A review of the literature shows that healthy activities of children and youths are highly dependent on the level of education of their parents: the higher the level of education, the greater the awareness of their parents of the role of physical activity in one's life [13, 14, 15, 16, 17]. Moreover, there are other important socio-economic factors: parents' profession which translates into income, family structure and place of residence [18, 19, 20, 21]. 
The aim of the research was the identification of chosen social variables that influence the physical activity of urban children and youths from the Bialskie district.

\section{Material and methods}

\section{Choice of trial}

The research was conducted on children and youths aged 1018 attending urban primary, secondary and high schools in Biała Podlaska and Międzyrzec Podlaski. School authorities, parents and guardians of the respondents agreed to take part in the research. About $35 \%$ of parents did not give permission to test their children. During the conduct of the project $10 \%$ of children refused to participate in the research, and 16\% of children did not participate due to absence from school. Ultimately, 1.084 students participated in the project, including 582 boys and 502 girls (tab. 1). The research was conducted by employees of the Faculty of Physical Education and Sport in Biała Podlaska as a part of statutory research DS.116, after the approval of Ethics Committee of the University of Physical Education in Warsaw.

Table 1. Numerical and percentage distribution of respondent boys and girls divided into age categories

\begin{tabular}{|c|c|c|c|c|}
\hline \multirow{2}{*}{$\begin{array}{c}\text { Age } \\
\text { categories }\end{array}$} & \multicolumn{2}{|c|}{ Boys } & \multicolumn{2}{c|}{ Girls } \\
\cline { 2 - 5 } & $\mathbf{n}$ & $\%$ & $\mathbf{n}$ & $\%$ \\
\hline $10-12$ & 206 & 35.4 & 198 & 39.4 \\
\hline $13-15$ & 162 & 27.8 & 142 & 28.3 \\
\hline $16-18$ & 214 & 36.8 & 162 & 32.3 \\
\hline Total & 582 & 100.0 & 502 & 100.0 \\
\hline
\end{tabular}

\section{Calendar age}

This was calculated from the difference between the date of research and the date of birth, changing the months and days to decimal parts of a year. This allowed classification of the researched people in the correct age group. For example children whose calendar age was between 11.50 to 12.49 were classified as 12-year-olds.

Respondents were divided into three age groups: 10 to 12,13 to 15 and 16 to 18 , which corresponded to the following levels of education: primary, lower secondary and secondary.

\section{Diagnostic survey}

Information about the participation of the respondents in Physical Education classes and structured extra-curricular sport activities, as well as about preferred forms of physical activity in free time, was gained via the diagnostic survey method with the use of the author's survey. The same method was used while assessing the socio-economic situation of respondents' families. The survey included basic personal data such as date of birth, sex of the respondents, number of children in the family, parents' education, profession and income per member of the family. Surveys were filled in by parents or guardians of the respondents.

\section{Division into groups based on the socio-economic \\ situation of the family}

Within three age groups i.e. 10 to 12,13 to 15 and 16 to 18 (separately for girls and boys), two groups were isolated: with higher and lower family socio-economic status, wherein every one of those factors was considered separately. And so respondents from families with an average or higher level of education were attributed to a higher status group, and those from families with basic and vocational education were attributed to a lower status group. In the context of the number of children in a family respondents from families with one or two children were attributed to the first (higher) group and those from families with three or more children were attributed to the second group.

Taking into account the type of work performed by the parents the first group included those respondents whose parents perform intellectual work, and the second group consisted of children of farmers and physical workers. During the analysis of income per family member, children from families with incomes higher than 1000 PLN per person were attributed to the higher status group and those from families with income lower than that were attributed to the lower status group.

\section{Statistical methods}

In order to analyze the relationship between the participation in structured extra-curricular physical activities and socioeconomic factors, the multifactoral logistic regression model was used [22]. In this model the differences between groups of different socio-economic status were assessed by the application of surplus ratio calculated as odds ratio (OR). The model shows the relations between the odds of occurrence of an event in a non-reference group (in this case the 'chance' of being active) in terms of occurrence of an event in the reference group. A ratio of surplus $\mathrm{OR}=1$ signifies a lack of relationship between the studied factor and an event, OR $>1$ means that the chance of occurrence of an event in the non-reference group is higher than in the reference group, while $\mathrm{OR}<1$ means that the chance of occurrence of an event in the non-reference group is lower than in the reference group. In the author's research the group of the lowermost socio-economic status was always the reference group.

In order to qualify the variability of analyzed characteristics within a group, the confidence interval (CI) was set at 95\%. The statistical relevance of the calculated odds ratio was assessed by the Wald test. The aforementioned calculations were made with the use of the Statistica 10 (StatSoft, Tulsa OK, USA) computer program.

\section{Results}

Analysis of the collected results showed that almost all respondents (over 95\%) participated in obligatory Physical Education classes. Only in the oldest group of girls (16-18) was the percentage of participants slightly lower (tab. 2).

Table 2. Participation in physical education classes according to gender and age

\begin{tabular}{|c|c|c|c|c|c|c|c|c|}
\hline \multirow{2}{*}{$\begin{array}{c}\text { Age } \\
\text { categories }\end{array}$} & \multicolumn{4}{|c|}{ Boys } & \multicolumn{4}{c|}{ Girls } \\
\cline { 2 - 9 } & \multicolumn{2}{|c|}{ Yes } & \multicolumn{2}{c|}{ No } & \multicolumn{2}{c|}{ Yes } & \multicolumn{2}{c|}{ No } \\
\cline { 2 - 9 } & $\mathbf{n}$ & $\%$ & $\mathbf{n}$ & $\%$ & $\mathbf{n}$ & $\%$ & $\mathbf{n}$ & $\%$ \\
\hline $10-12$ & 198 & 96.1 & 8 & 3.9 & 192 & 97.0 & 6 & 3.0 \\
\hline $13-15$ & 161 & 99.4 & 1 & 0.6 & 140 & 98.6 & 2 & 1.4 \\
\hline $16-18$ & 212 & 99.1 & 2 & 0.9 & 150 & 92.6 & 12 & 7.4 \\
\hline Total & 571 & 98.1 & 11 & 1.9 & 482 & 96.0 & 20 & 4.0 \\
\hline
\end{tabular}

The collected data shows, that over half of the respondents participated in structured extra-curricular physical activities (62.7\% of boys and $56 \%$ of girls). Those classes were a part of School Sports Clubs, sports clubs or other structured forms of physical activity (e.g. swimming classes, horse riding, karate, etc.).

In the oldest girls group the percentage of the respondents that participated in those classes was much lower than in other age groups - only one third of the respondents declared participation in those activities (tab. 3). 
Table 3. Participation in extra-curricular physical activities according to gender and age

\begin{tabular}{|c|c|c|c|c|c|c|c|c|}
\hline \multirow{2}{*}{$\begin{array}{c}\text { Age } \\
\text { categories }\end{array}$} & \multicolumn{4}{|c|}{ Boys } & \multicolumn{4}{c|}{ Girls } \\
\cline { 2 - 9 } & \multicolumn{2}{|c|}{ Yes } & \multicolumn{2}{c|}{ No } & \multicolumn{2}{c|}{ Yes } & \multicolumn{2}{c|}{ No } \\
\cline { 2 - 9 } & $\mathbf{n}$ & $\%$ & $\mathbf{n}$ & $\%$ & $\mathbf{n}$ & $\%$ & $\mathbf{n}$ & $\%$ \\
\hline $10-12$ & 139 & 67.5 & 67 & 32.5 & 133 & 66.8 & 66 & 33.2 \\
\hline $13-15$ & 105 & 64.8 & 57 & 35.2 & 94 & 65.7 & 49 & 34.3 \\
\hline $16-18$ & 121 & 56.5 & 93 & 43.5 & 54 & 33.8 & 106 & 66.3 \\
\hline Total & 365 & 62.7 & 217 & 37.3 & 281 & 56.0 & 221 & 44.0 \\
\hline
\end{tabular}

Factors that are most often used in a socio-economic status assessment are parents' education, number of children in a family, parents' profession, and income. While analyzing the influence of parents' education and the number of children in a family on participation in structured physical activities, a distinctive relationship between observed variables was not observed. Only in the case of fathers' education was surplus observed $(\mathrm{OR}=2.68)$ in the oldest girls group. This meant that girls whose fathers have average or higher education had almost 2.5 times more chance to be active than their female peers from families in which the fathers had basic or vocational education (tab. 4-6).

Table 4. Participation in extra-curricular physical activities of respondent boys and girls, depending on the number of children in the family

\begin{tabular}{|c|c|c|c|c|c|c|c|c|}
\hline \multirow{2}{*}{$\begin{array}{c}\text { Age } \\
\text { categories }\end{array}$} & \multirow{2}{*}{$\begin{array}{c}\text { Number } \\
\text { of children }\end{array}$} & \multicolumn{2}{|c|}{ Participate } & \multicolumn{2}{|c|}{ Not participate } & \multirow{2}{*}{ OR } & \multirow{2}{*}{$95 \% \mathrm{Cl}$} & \multirow[b]{2}{*}{$p$} \\
\hline & & $n$ & $\%$ & $n$ & $\%$ & & & \\
\hline \multicolumn{9}{|c|}{ Boys } \\
\hline \multirow{2}{*}{$\begin{array}{l}10-12 \\
\text { years }\end{array}$} & 1.2 & 72 & 66.1 & 37 & 33.9 & 0,77 & \multirow{2}{*}{$0.39-1.50$} & \multirow{2}{*}{0.596} \\
\hline & 3 or more & 48 & 71.6 & 19 & 28.4 & 1 & & \\
\hline \multirow{2}{*}{$\begin{array}{l}13-15 \\
\text { years }\end{array}$} & 1.2 & 61 & 64.2 & 34 & 35.8 & 0,83 & \multirow{2}{*}{$0.41-1.67$} & \multirow{2}{*}{0.597} \\
\hline & 3 or more & 39 & 68.4 & 18 & 31.6 & 1 & & \\
\hline \multirow{2}{*}{$\begin{array}{l}16-18 \\
\text { years }\end{array}$} & 1.2 & 40 & 54.8 & 33 & 45.2 & 0,91 & \multirow{2}{*}{$0.51-1.62$} & \multirow{2}{*}{0.756} \\
\hline & 3 or more & 77 & 57.0 & 58 & 43.0 & 1 & & \\
\hline \multicolumn{9}{|c|}{ Girls } \\
\hline \multirow{2}{*}{$\begin{array}{l}10-12 \\
\text { years }\end{array}$} & 1.2 & 83 & 68.6 & 38 & 31.4 & 1,22 & \multirow{2}{*}{$0.64-2.33$} & \multirow{2}{*}{0.533} \\
\hline & 3 or more & 41 & 64.1 & 23 & 35.9 & 1 & & \\
\hline \multirow{2}{*}{$\begin{array}{l}13-15 \\
\text { years }\end{array}$} & 1.2 & 51 & 66.2 & 26 & 33.8 & 1,06 & \multirow{2}{*}{$0.52-2.16$} & \multirow{2}{*}{0.88} \\
\hline & 3 or more & 39 & 65.0 & 21 & 35.0 & 1 & & \\
\hline \multirow{2}{*}{$\begin{array}{l}16-18 \\
\text { years }\end{array}$} & 1.2 & 20 & 33.9 & 39 & 66.1 & 1,10 & \multirow{2}{*}{$0.54-2.23$} & \multirow{2}{*}{0.792} \\
\hline & 3 or more & 28 & 31.8 & 60 & 68.2 & 1 & & \\
\hline
\end{tabular}

OR - odds ratio,

CI - confidence interval,

$\mathrm{p}$ - level of relevance,

* - difference statistically significant at $\mathrm{p}<0.05$.
Table 5. Participation in extra-curricular physical activities of surveyed boys and girls according to father's education

\begin{tabular}{|c|c|c|c|c|c|c|c|c|}
\hline \multirow{2}{*}{$\begin{array}{c}\text { Age } \\
\text { categories }\end{array}$} & \multirow{2}{*}{$\begin{array}{l}\text { Father's } \\
\text { education }\end{array}$} & \multicolumn{2}{|c|}{ Participate } & \multicolumn{2}{|c|}{ Not participate } & \multirow{2}{*}{ OR } & \multirow{2}{*}{$95 \% \mathrm{Cl}$} & \multirow{2}{*}{$p$} \\
\hline & & $n$ & $\%$ & $n$ & $\%$ & & & \\
\hline \multicolumn{9}{|c|}{ Boys } \\
\hline \multirow{2}{*}{$\begin{array}{l}10-12 \\
\text { years }\end{array}$} & $\begin{array}{c}\text { basic }+ \\
\text { vocational }\end{array}$ & 55 & 69.6 & 24 & 30.4 & 1 & \multirow{2}{*}{$0.47-1.69$} & \multirow{2}{*}{0.712} \\
\hline & $\begin{array}{c}\text { average + } \\
\text { higher }\end{array}$ & 65 & 67.0 & 32 & 33.0 & 0.89 & & \\
\hline \multirow{2}{*}{$\begin{array}{l}13-15 \\
\text { years }\end{array}$} & $\begin{array}{c}\text { basic }+ \\
\text { vocational }\end{array}$ & 44 & 71.0 & 18 & 29.0 & 1 & \multirow{2}{*}{$0.34-1.36$} & \multirow{2}{*}{0.270} \\
\hline & $\begin{array}{c}\text { average + } \\
\text { higher }\end{array}$ & 58 & 62.4 & 35 & 37.6 & 0.68 & & \\
\hline \multirow{2}{*}{$\begin{array}{l}16-18 \\
\text { years }\end{array}$} & $\begin{array}{c}\text { basic }+ \\
\text { vocational }\end{array}$ & 76 & 53.5 & 66 & 46.5 & 1 & \multirow{2}{*}{$0.78-2.60$} & \multirow{2}{*}{0.245} \\
\hline & $\begin{array}{c}\text { average + } \\
\text { higher }\end{array}$ & 41 & 62.1 & 25 & 37.9 & 1.42 & & \\
\hline \multicolumn{9}{|c|}{ Girls } \\
\hline \multirow{2}{*}{$\begin{array}{l}10-12 \\
\text { years }\end{array}$} & $\begin{array}{c}\text { basic }+ \\
\text { vocational }\end{array}$ & 51 & 68.9 & 23 & 31.1 & 1 & \multirow{2}{*}{$0.46-1.63$} & \multirow{2}{*}{0.655} \\
\hline & $\begin{array}{l}\text { average + } \\
\text { higher }\end{array}$ & 73 & 65.8 & 38 & 34.2 & 0.87 & & \\
\hline \multirow{2}{*}{$\begin{array}{l}13-15 \\
\text { years }\end{array}$} & $\begin{array}{c}\text { basic }+ \\
\text { vocational }\end{array}$ & 35 & 61.4 & 22 & 38.6 & 1 & \multirow{2}{*}{$0.67-2.84$} & \multirow{2}{*}{0.373} \\
\hline & $\begin{array}{c}\text { average + } \\
\text { higher }\end{array}$ & 55 & 68.8 & 25 & 31.3 & 1.38 & & \\
\hline \multirow{2}{*}{$\begin{array}{l}16-18 \\
\text { years }\end{array}$} & $\begin{array}{c}\text { basic }+ \\
\text { vocational }\end{array}$ & 22 & 24.4 & 68 & 75.6 & 1 & \multirow{2}{*}{$1.31-5.49$} & \multirow{2}{*}{0.007} \\
\hline & $\begin{array}{c}\text { average + } \\
\text { higher }\end{array}$ & 26 & 46.4 & 30 & 53.6 & 2.68 & & \\
\hline
\end{tabular}

OR - odds ratio,

$\mathrm{CI}$ - confidence interval,

$\mathrm{p}$ - level of relevance,

* - difference statistically significant at $\mathrm{p}<0.05$.

Table 6. Participation in extra-curricular physical activities of surveyed boys and girls according to mother's education

\begin{tabular}{|c|c|c|c|c|c|c|c|c|}
\hline \multirow{2}{*}{$\begin{array}{c}\text { Age } \\
\text { categories }\end{array}$} & \multirow{2}{*}{$\begin{array}{l}\text { Mother's } \\
\text { education }\end{array}$} & \multicolumn{2}{|c|}{ Participate } & \multicolumn{2}{|c|}{ Not participate } & \multirow{2}{*}{$O R$} & \multirow{2}{*}{$95 \%$ Cl } & \multirow{2}{*}{$p$} \\
\hline & & $n$ & $\%$ & $n$ & $\%$ & & & \\
\hline \multicolumn{9}{|c|}{ Boys } \\
\hline \multirow{2}{*}{$\begin{array}{l}10-12 \\
\text { years }\end{array}$} & $\begin{array}{c}\text { basic }+ \\
\text { vocational }\end{array}$ & 35 & 64.8 & 19 & 35.2 & 1 & \multirow{2}{*}{$0.63-2.47$} & \multirow{2}{*}{0.524} \\
\hline & $\begin{array}{c}\text { average + } \\
\text { higher }\end{array}$ & 85 & 69.7 & 37 & 30.3 & 1.25 & & \\
\hline \multirow{2}{*}{$\begin{array}{l}13-15 \\
\text { years }\end{array}$} & $\begin{array}{c}\text { basic }+ \\
\text { vocational }\end{array}$ & 23 & 59.0 & 16 & 41.0 & 1 & \multirow{2}{*}{$0.72-3.27$} & \multirow[b]{2}{*}{0.268} \\
\hline & $\begin{array}{c}\text { average + } \\
\text { higher }\end{array}$ & 77 & 68.8 & 35 & 31.3 & 1.53 & & \\
\hline \multirow{2}{*}{$\begin{array}{l}16-18 \\
\text { years }\end{array}$} & $\begin{array}{c}\text { basic }+ \\
\text { vocational }\end{array}$ & 58 & 54.2 & 49 & 45.8 & 1 & \multirow{2}{*}{$0.68-2.06$} & \multirow{2}{*}{0.541} \\
\hline & $\begin{array}{l}\text { average + } \\
\text { higher }\end{array}$ & 59 & 58.4 & 42 & 41.6 & 1.19 & & \\
\hline \multicolumn{9}{|c|}{ Girls } \\
\hline \multirow{2}{*}{$\begin{array}{l}10-12 \\
\text { years }\end{array}$} & $\begin{array}{c}\text { basic }+ \\
\text { vocational }\end{array}$ & 31 & 67.4 & 15 & 32.6 & 1 & \multirow{2}{*}{$0.48-1.99$} & \multirow{2}{*}{0.951} \\
\hline & $\begin{array}{l}\text { average + } \\
\text { higher }\end{array}$ & 93 & 66.9 & 46 & 33.1 & 0.98 & & \\
\hline \multirow{2}{*}{$\begin{array}{l}13-15 \\
\text { years }\end{array}$} & $\begin{array}{c}\text { basic }+ \\
\text { vocational }\end{array}$ & 23 & 56.1 & 18 & 43.9 & 1 & \multirow{2}{*}{$0.84-3.87$} & \multirow{2}{*}{0.124} \\
\hline & $\begin{array}{c}\text { average + } \\
\text { higher }\end{array}$ & 67 & 69.8 & 29 & 30.2 & 1.81 & & \\
\hline \multirow{2}{*}{$\begin{array}{l}16-18 \\
\text { years }\end{array}$} & $\begin{array}{c}\text { basic }+ \\
\text { vocational }\end{array}$ & 22 & 29.7 & 52 & 70.3 & 1 & \multirow{2}{*}{$0.65-2.63$} & \multirow{2}{*}{0.447} \\
\hline & $\begin{array}{c}\text { average + } \\
\text { higher }\end{array}$ & 26 & 35.6 & 47 & 64.4 & 1.31 & & \\
\hline
\end{tabular}

OR - odds ratio,

CI - confidence interval,

$\mathrm{p}$ - level of relevance,

* - difference statistically significant at $\mathrm{p}<0.05$. 
In the case of respondents' mothers' and fathers' professions a higher participation in structured extra-curricular physical activities by boys and girls whose parents performed intellectual work was observed. Only in the group of 13- to 15-year-olds (both girls and boys) was there a reverse relationship in the case of profession held by the father. Those who had a father that worked as a farmer or physical worker were more active. However, the values of the odds ratios were not statistically relevant except for the oldest girls group in the context of mother's profession (tab. 7 and 8).

Table 7. Participation in extra-curricular physical activities of surveyed boys and girls according to father's profession

\begin{tabular}{|c|c|c|c|c|c|c|c|c|}
\hline \multirow{2}{*}{$\begin{array}{c}\text { Age } \\
\text { categories }\end{array}$} & \multirow{2}{*}{$\begin{array}{l}\text { Father's } \\
\text { profession }\end{array}$} & \multicolumn{2}{|c|}{ Participate } & \multicolumn{2}{|c|}{ Not participate } & \multirow{2}{*}{ OR } & \multirow{2}{*}{$95 \% \mathrm{CI}$} & \multirow[b]{2}{*}{$p$} \\
\hline & & $n$ & $\%$ & $n$ & $\%$ & & & \\
\hline \multicolumn{9}{|c|}{ Boys } \\
\hline \multirow{2}{*}{$\begin{array}{l}10-12 \\
\text { years }\end{array}$} & $\begin{array}{c}\text { farmer+ } \\
\text { physical labour }\end{array}$ & 14 & 60.9 & 9 & 39.1 & 1 & \multirow{2}{*}{$0.57-3.54$} & \multirow{2}{*}{0.446} \\
\hline & \begin{tabular}{|l|} 
self employed+ \\
intellectual work
\end{tabular} & 104 & 68.9 & 47 & 31.1 & 1.42 & & \\
\hline \multirow{2}{*}{$\begin{array}{l}13-15 \\
\text { years }\end{array}$} & \begin{tabular}{|c|} 
farmer + \\
physical labour \\
\end{tabular} & 13 & 72.2 & 5 & 27.8 & 1 & \multirow[b]{2}{*}{$0.24-2.14$} & \multirow{2}{*}{0.541} \\
\hline & \begin{tabular}{|c|} 
self employed + \\
intellectual work
\end{tabular} & 87 & 64.9 & 47 & 35.1 & 0.71 & & \\
\hline \multirow{2}{*}{$\begin{array}{l}16-18 \\
\text { years }\end{array}$} & \begin{tabular}{c|} 
farmer + \\
physical labour
\end{tabular} & 37 & 47.4 & 41 & 52.6 & 1 & \multirow{2}{*}{$0.99-3.10$} & \multirow{2}{*}{0.053} \\
\hline & \begin{tabular}{|c|} 
self employed + \\
intellectual work
\end{tabular} & 79 & 61.2 & 50 & 38.8 & 1.75 & & \\
\hline \multicolumn{9}{|c|}{ Girls } \\
\hline \multirow{2}{*}{$\begin{array}{l}10-12 \\
\text { years }\end{array}$} & \begin{tabular}{c|} 
farmer + \\
physical labour
\end{tabular} & 18 & 62.1 & 11 & 37.9 & 1 & \multirow{2}{*}{$0.58-3.03$} & \multirow{2}{*}{0.506} \\
\hline & \begin{tabular}{|c|} 
self employed + \\
intellectual work
\end{tabular} & 106 & 68.4 & 49 & 31.6 & 1.32 & & \\
\hline \multirow{2}{*}{$\begin{array}{l}13-15 \\
\text { years }\end{array}$} & \begin{tabular}{c|} 
farmer + \\
physical labour
\end{tabular} & 17 & 70.8 & 7 & 29.2 & 1 & \multirow{2}{*}{$0.28-1.98$} & \multirow{2}{*}{0.560} \\
\hline & \begin{tabular}{|c|} 
self employed + \\
intellectual work
\end{tabular} & 73 & 64.6 & 40 & 35.4 & 0.75 & & \\
\hline \multirow{2}{*}{$\begin{array}{l}16-18 \\
\text { years }\end{array}$} & \begin{tabular}{c|} 
farmer + \\
physical labour
\end{tabular} & 15 & 30.0 & 35 & 70.0 & 1 & \multirow{2}{*}{$0.57-2.53$} & \multirow{2}{*}{0.622} \\
\hline & \begin{tabular}{|c|} 
self employed + \\
intellectual work
\end{tabular} & 33 & 34.0 & 64 & 66.0 & 1.2 & & \\
\hline
\end{tabular}

OR - odds ratio,

CI - confidence interval,

$\mathrm{p}$ - level of relevance,

* - difference statistically significant at $\mathrm{p}<0.05$.
Table 8. Participation in extra-curricular physical activities of surveyed boys and girls according to mother's profession

\begin{tabular}{|c|c|c|c|c|c|c|c|c|}
\hline \multirow{2}{*}{$\begin{array}{c}\text { Age } \\
\text { categories }\end{array}$} & \multirow{2}{*}{$\begin{array}{l}\text { Mother's } \\
\text { profession }\end{array}$} & \multicolumn{2}{|c|}{ Participate } & \multicolumn{2}{|c|}{ Not participate } & \multirow{2}{*}{$\mathrm{OR}$} & \multirow{2}{*}{$95 \%$ Cl } & \multirow{2}{*}{$p$} \\
\hline & & $n$ & $\%$ & $n$ & $\%$ & & & \\
\hline \multicolumn{9}{|c|}{ Boys } \\
\hline \multirow{2}{*}{$\begin{array}{l}10-12 \\
\text { years }\end{array}$} & \begin{tabular}{|c|} 
farmer + \\
physical labour
\end{tabular} & 28 & 63.6 & 16 & 36.4 & 1 & \multirow{2}{*}{$0.63-2.68$} & \multirow{2}{*}{0.474} \\
\hline & \begin{tabular}{|c|} 
self employed + \\
intellectual work
\end{tabular} & 91 & 69.5 & 40 & 30.5 & 1.30 & & \\
\hline \multirow{2}{*}{$\begin{array}{l}13-15 \\
\text { years }\end{array}$} & \begin{tabular}{c|} 
farmer + \\
physical labour
\end{tabular} & 29 & 61.7 & 18 & 38.3 & 1 & \multirow{2}{*}{$0.63-2.67$} & \multirow{2}{*}{0.478} \\
\hline & $\begin{array}{c}\text { self employed + } \\
\text { intellectual work }\end{array}$ & 71 & 67.6 & 34 & 32.4 & 1.30 & & \\
\hline \multirow{2}{*}{$\begin{array}{l}16-18 \\
\text { years }\end{array}$} & $\begin{array}{c}\text { farmer + } \\
\text { physical labour }\end{array}$ & 45 & 49.5 & 46 & 50.5 & 1 & \multirow{2}{*}{$0.93-2.86$} & \multirow{2}{*}{0.083} \\
\hline & \begin{tabular}{|c|} 
self employed + \\
intellectual work
\end{tabular} & 72 & 61.5 & 45 & 38.5 & 1.64 & & \\
\hline \multicolumn{9}{|c|}{ Girls } \\
\hline \multirow{2}{*}{$\begin{array}{l}10-12 \\
\text { years }\end{array}$} & $\begin{array}{c}\text { farmer }+ \\
\text { physical labour }\end{array}$ & 26 & 55.3 & 21 & 44.7 & 1 & \multirow{2}{*}{$0.97-3.86$} & \multirow{2}{*}{0.058} \\
\hline & \begin{tabular}{|c|} 
self employed + \\
intellectual work
\end{tabular} & 96 & 70.6 & 40 & 29.4 & 1.94 & & \\
\hline \multirow{2}{*}{$\begin{array}{l}13-15 \\
\text { years }\end{array}$} & $\begin{array}{c}\text { farmer + } \\
\text { physical labour }\end{array}$ & 16 & 53.3 & 14 & 46.7 & 1 & \multirow{2}{*}{$0.85-4.52$} & \multirow{2}{*}{0.110} \\
\hline & \begin{tabular}{|c|} 
self employed + \\
intellectual work
\end{tabular} & 74 & 69.2 & 33 & 30.8 & 1.96 & & \\
\hline \multirow{2}{*}{$\begin{array}{l}16-18 \\
\text { years }\end{array}$} & \begin{tabular}{|c|} 
farmer + \\
physical labour
\end{tabular} & 12 & 21.8 & 43 & 78.2 & 1 & \multirow{2}{*}{$1.06-4.98$} & \multirow{2}{*}{0.032} \\
\hline & \begin{tabular}{|c|} 
self employed + \\
intellectual work
\end{tabular} & 36 & 39.1 & 56 & 60.9 & 2.3 & & \\
\hline
\end{tabular}

OR - odds ratio,

$\mathrm{CI}$ - confidence interval,

$\mathrm{p}$ - level of relevance,

* - difference statistically significant at $\mathrm{p}<0.05$.

It was shown that participation in structured extra-curricular physical activities was dependent on income per family member. It was found that participation in those activities was higher in the respondents from families with incomes higher that 1000 PLN per family member. This relationship occurred in both girls and boys from all analyzed age groups. Analysis of the odds ratio showed that the OR coefficient was high and statistically relevant in groups of 10- to 12-year-old boys and 16- to 18-year-old girls. (tab. 9). 
Table 9. Participation in extra-curricular physical activities of surveyed boys and girls according to income

\begin{tabular}{|c|c|c|c|c|c|c|c|c|}
\hline \multirow{2}{*}{$\begin{array}{c}\text { Age } \\
\text { categories }\end{array}$} & \multirow{2}{*}{ Income } & \multicolumn{2}{|c|}{ Participate } & \multicolumn{2}{|c|}{ Not participate } & \multirow{2}{*}{ OR } & \multirow{2}{*}{$95 \%$ Cl } & \multirow{2}{*}{ p } \\
\hline & & $n$ & $\%$ & $n$ & $\%$ & & & \\
\hline \multicolumn{9}{|c|}{ Boys } \\
\hline \multirow{2}{*}{$\begin{array}{l}10-12 \\
\text { years }\end{array}$} & up to 1000 PLN & 91 & 65.5 & 48 & 34.5 & 1 & \multirow{2}{*}{$0.73-5.13$} & \multirow{2}{*}{0.047} \\
\hline & \begin{tabular}{|l|} 
over 1000 PLN \\
\end{tabular} & 29 & 80.5 & 7 & 19.5 & 2.19 & & \\
\hline \multirow{2}{*}{\begin{tabular}{l|}
$13-15$ \\
years
\end{tabular}} & up to 1000 PLN & 69 & 62.7 & 41 & 37.3 & 1 & \multirow{2}{*}{$0.51-3.05$} & \multirow{2}{*}{0.107} \\
\hline & \begin{tabular}{|l|} 
over 1000 PLN \\
\end{tabular} & 31 & 73.8 & 11 & 26.2 & 1.68 & & \\
\hline \multirow{2}{*}{\begin{tabular}{l|}
$16-18$ \\
years
\end{tabular}} & up to 1000 PLN & 101 & 56.1 & 79 & 43.9 & 1 & \multirow{2}{*}{$0.32-1.91$} & \multirow{2}{*}{0.217} \\
\hline & over 1000 PLN & 16 & 57.1 & 12 & 42.9 & 1.13 & & \\
\hline \multicolumn{9}{|c|}{ Girls } \\
\hline \multirow{2}{*}{$\begin{array}{l}10-12 \\
\text { years }\end{array}$} & up to $1000 \mathrm{PLN}$ & 93 & 64.1 & 52 & 35.9 & 1 & \multirow{2}{*}{$0.79-6.36$} & \multirow{2}{*}{0.38} \\
\hline & over 1000 PLN & 31 & 77.5 & 9 & 22.5 & 1.92 & & \\
\hline \multirow{2}{*}{$\begin{array}{l}13-15 \\
\text { years }\end{array}$} & up to 1000 PLN & 62 & 62.6 & 37 & 37.4 & 1 & \multirow{2}{*}{$0.72-4.86$} & \multirow{2}{*}{0.191} \\
\hline & over 1000 PLN & 28 & 73.7 & 10 & 26.3 & 1.68 & & \\
\hline \multirow{2}{*}{$\begin{array}{l}16-18 \\
\text { years }\end{array}$} & up to 1000 PLN & 36 & 28.3 & 91 & 71.7 & 1 & \multirow{2}{*}{ 1.14-8.72 } & \multirow{2}{*}{$0.025^{*}$} \\
\hline & \begin{tabular}{|l|} 
over 1000 PLN \\
\end{tabular} & 12 & 60.0 & 8 & 40.0 & 3.85 & & \\
\hline
\end{tabular}

OR - odds ratio,

$\mathrm{CI}$ - confidence interval,

$\mathrm{p}$ - level of relevance,

* - difference statistically significant at $\mathrm{p}<0.05$.

In the case of respondents who were physically active, participation in other non-targeted forms of physical activity was taken into account, depending on family income, because this variable is the most differentiating as regards participation in structured extra-curricular physical activities.

The conducted research shows that, irrespective of income per family member, the most popular non-targeted forms of physical activities were cycling and football (except for the youngest group of girls, where, except for cycling, the most popular form of physical activity was physical play with peers).

Income, however, did influence the frequency of choice by the respondents of such forms of physical activities as winter sports and swimming - those forms of activities were mostly chosen by people from families with higher incomes per family member (fig. 1 and 2).

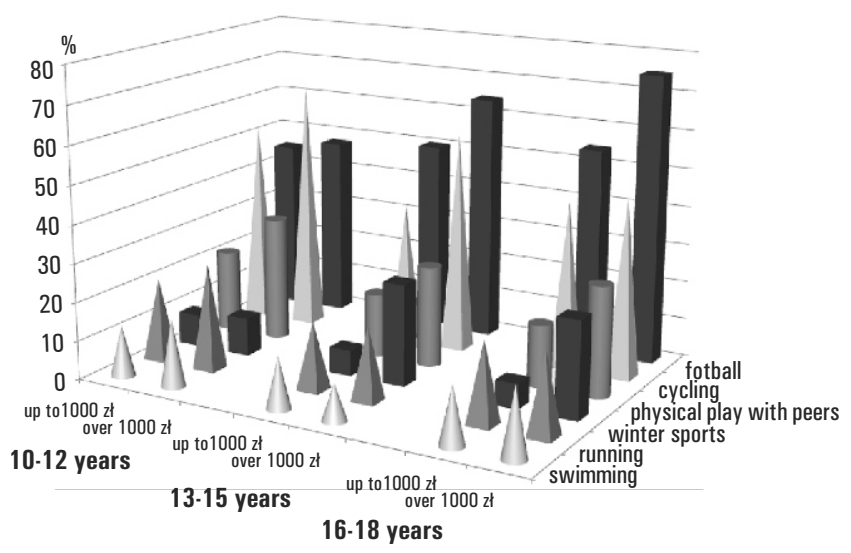

Figure 1. Preferred forms of unstructured physical activity in boys according to the financial situation of the family

(there was a choice of several answers)

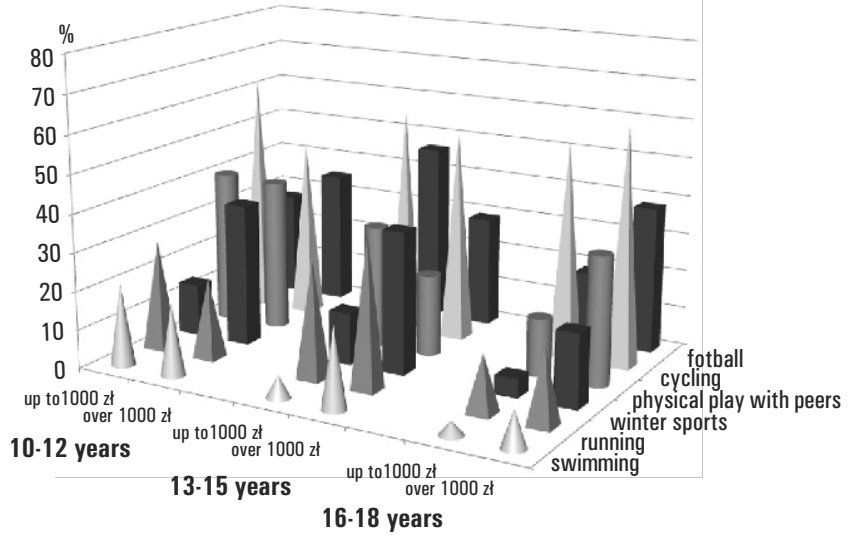

Figure 2. Preferred forms of non-structured physical activity in girls according to the financial situation of the family

(there was a choice of several answers)

\section{Discussion}

The importance of certain socio-economic factors in differentiation of children's and youth's physical activities keeps changing. In previous studies the relationship between parents' education and their children's physical activity was often underlined. Most studies show that a higher level of parents' education was connected with higher children's physical activity compared to their peers from families in which mothers and fathers had lower levels of education [15, 23, 24]. In the author's research this relationship was not observed.

Parents' education did not affect the participation of their children in structured extra-curricular physical activities significantly. This is also confirmed by studies conducted by Mańczak and Raciborski [25], and Ortlieb et al. [26]. This could be explained by the fact that nowadays parents' education is more of a carrier of cultural than economic values and physical activity is strongly connected with families' financial resources.

Another socio-economic variable analyzed in the research (size of the family) also did not show a connection with structured extra-curricular physical activities. However, in the literature, opinions on the influence of this factor on participation in targeted physical activity are divided. Bagley et al. [27] and Cleland et al. [28] showed that boys and girls who have siblings are more physically active. On the other hand in studies conducted by Miązek et al. [29], Pastuszak [15], and Ortlieb et al. [26] there were no statistically relevant relationships between analyzed variables. It could be assumed that this variable will influence physical activity less and less, because nowadays fertility in families is decreasing: families with five or more children are rare.

The income of the families of children that took part in the research turned out to be the most important factor in differentiating the participation in structured physical activities. Information on the material situation of families was very hard to obtain, which may have been caused by cultural, economic and legal reasons. Because of this there are rare reports that connect this variable with physical activity. In the research conducted by Kantomaa et al. [30], the occurrence of a positive relationship between physical activity and the income of families of Finnish youths was observed. A similar correlation was observed in research conducted on Italian children [31]. In the research conducted by Federico et al. [31] it was observed that the odds ratio showed greater physical activity of children from families with a higher occupational category (intellectual workers) in comparison to respondents who had at least one unemployed parent. 
The good economic situation of the family affords the possibility of attending different types of sports facilities or participating in different forms of leisure activity. Among the sports that require significant financial resources are tennis, skiing, ice skating and swimming. Costs are related to, for example, the purchase or hire of equipment, payment for the use of a sports facility, or even a trip to another town. Whereas in other forms of physical activity such as football or running, the costs connected to those sports are much lower. Conducted research observed that people from families with higher incomes per person more often chose such forms of physical activity as winter sports and swimming. Similar observations were shown in studies by Lewandowska et al. [32] in which the conditioning of forms of physical activities by children from Warsaw was analyzed. This research showed that there was a higher attendance rate of girls and boys from families with parents with higher education in extra-curricular activities such as swimming, tennis, horse riding, martial arts and strength sports. Those were mostly fee-carrying classes. Whereas children from families with lower education (basic or vocational) more often participated in extra-curricular activities organized in schools.

In conclusion one may assume that as a result of the further functioning of the market economy and increasing globalization the differences in income will increase but the standards of living of the poorest families will improve only gradually, which, sooner or later, will probably lead to the disappearance of social gradients. It may be informed most of all by parents' education and number of children in a family. Over the last few years a depreciation of higher education on the labor market has been observed. Moreover, families with a lot of children are increasingly rare.

\section{Conclusions}

1. The overwhelming majority of respondents participated in physical education classes, and more than half in extra-curricular physical activities.

2. A distinctive relationship between parents' education, the number of children in a family and attendance in structured physical activities was not observed. A higher attendance rate of girls and boys whose parents' were intellectual workers was observed.

3. Increasing disproportions in societal wealth may be an important factor limiting the participation of the poorest group of children and youths in targeted physical activity.

4. A lack of distinctive effect on physical activities of such socio-economic variables as parents' education and the number of children is probably connected with the change of importance of such factors in modern society (depreciation of the role of higher education, decrease in fertility).

\section{Literature}

1. Andersen L.B., Harro M., Sardinha L.B., Froberg K., Ekelund U., Brage S. et al. (2006). Physical activity and clustered cardiovascular risk in children: a cross-sectional study (The European Youth Heart Study). Lancet 368, 299-304.

2. Raczyńska B., Długołęcka B. (2009). Physical activity in osteoporosis prevention and treatment. Polish Journal of Sport and Tourism 16(1), 1-14.

3. Shephard R.J. (2007). Response of brain, liver, kidney, and other organs and tissues to regular physical activity. In C. Bouchard, S.N. Blair, W.L. Haskell (eds), Physical activity and health (pp. 127-140). Chapmaign IL: Human Kinetics.
4. Stoedefalke K., Amstrong N., Kirby B.J., Welsman J.R. (2000). Effect of training on peak oxygen uptake and blood lipids in 13- to 14-year-old girls. Acta Paediatrica 89, 12901294.

5. Annesi J.J. (2005). Improvements in self-concept associated with reduction on negative mood in preadolescents enrolled in an after school physical activity program. Psychological Report 97, 400-404.

6. Lawson H.A. (2005). Empowering people, facilitating community development, and contributing to sustainable development: the social work of sport, exercise and physical education programs. Sport Education and Society 10, 135-160.

7. World Health Organization. (2008). Promoting active living in and trough schools: a WHO statement. Geneva: WHO Press.

8. TNS Opinion \& Social. (2009). Sport and Physical Activity. Eurobarometer 72.3. Brussels: European Commission.

9. Currie C., Zanotti C., Morgan A., Currie D., de Looze M., Roberts Ch. et al. (2012). Social determinants of health and well-being among young people. Health Behavior in Schoolaged Children (HBSC) Study: international report from the 2009/2010 survey. Health Policy for Children and Adolescents 6, 129-132.

10. Mattocks C., Deere K., Leary S., Ness A., Tiling K., Blair S.N. et al. (2008). Early life determinants of physical activity in 11- to 12-years-olds: cohort study. British Journal of Sport Medicine 42(9), 721-724.

11. Hendrie G.A., Coveney J., Cox D.N. (2011). Factor analysis shows association between family activity environment and children's health behavior. Australian and New Zealand Journal of Public Health 35(6), 524-529.

12. Fuemmeler B.E., Andersen C.B., Mâsse L.C. (2011). Parentchild relationship of directly measured physical activity. International Journal of Behavioral Nutrition and Physical Activity 8, 17.

13. Borodulin K., Laatikainen T., Lahti-Koski M., Jousilahti P., Lakka T.A. (2008). Association of age and education with different types of leisure time physical activity among 4437 Finnish adults. Journal of Physical Activity and Health 5, 242251.

14. Mäkinen T., Kestilä L., Borodulin K., Martelin T., Rahkonen O., Prättälä R. (2009). Effect of childhood socio-economic conditions on educational differences in leisure-time physical activity. European Journal of Public Health 20(3), 346353.

15. Pastuszak A. (2006). Social determinants of physical activity of children and adolescents. Przeglaqd Naukowy Kultury Fizycznej Uniwersytetu Rzeszowskiego 1, 25-32.

16. Tammelin T., Nayha S., Laitinen J., Rintamaki H., Jarvelin M.R. (2003). Physical activity and social status in adolescence as predictors of physical inactivity in adulthood. Preventive Medicine 37, 375-381.

17. Warde J., Steptoe A. (2003). Socioeconomic differences in attitudes and beliefs about healthy lifestyles. Journal of Epidemiology and Community Health 57, 440-443.

18. Bagley S., Salmon J., Crawford D. (2006). Family structure and children's television viewing and physical activity. Medicine \& Science in Sports \& Exercise 38, 910-918.

19. Brodersen N.H., Steptoe A., Boniface D., Wardle J. (2007). Trends in physical activity and sedentary behavior in adolescence: ethnic and socioeconomic differences. British Journal of Sports Medicine 41(3), 140-144.

20. Ozdirenc M., Akim A.O., Geleck N. (2005). Physical fitness in rural children compared with urban children in Turkey. Pediatrics International 47, 26-31.

21. Ziviani J., Wadley D., Ward H., Macdonald D., Jenkins D., Rodger S. (2008). A place to play: socioeconomic and spatial factors in children's physical activity. Australian Occupational Therapy Journal 55(1), 2-11. 
22. Hosmer D.W., Lemeshow S. (1989). Applied logistic regression. New York: John Wiley \& Sons.

23. Jurak G., Kovać M., Strel J. (2002). Differences in spending summer holidays of Slovenian children and youth in different periods of schooling. International Journal of Physical Education 39(2), 34-43.

24. Morgan C.F., MacKenzie T.L., Sallis J.F., Broyles S.L., Zive M.M., Nader P.R. (2003). Personal, social and environmental correlates of physical activity in bi-ethnic sample of adolescents. Pediatric Exercise Science 15, 288-301.

25. Mańczak M., Raciborski F. (2013). Determinants of physical activity of first grade students of primary schools in Warsaw. Problemy Higieny i Epidemiologii 94(1), 79-85. [in Polish]

26. Ortlieb S., Schneider G., Coletzko S., Berdel D., von Berg A., Bauer C.P. et al. (2013). Physical activity and its correlates in children: a cross-sectional study (the GINIplus \& LISAplus studies). BMC Public Health 13, 349-362.

27. Bagley S., Salmon J., Crawford D. (2006). Family structure and children's television viewing and physical activity. Medicine \& Science in Sports \& Exercise 38, 910-918.

28. Cleland V., Timperio A., Salmon J., Hume C., Telford A., Crawford D. (2011). A longitudinal study of the family physical activity environmental and physical activity among youth. American Journal of Health Promotion 25(3), 159-167.
29. Miązek U., Szyndera M., Żiżka-Salamon D. (2005). Associations of physical activity and socioeconomic relationship of women lifestyle who study in Krakow. Annales Universitatis Mariae Curie-Skłodowska, Sectio D LX, suppl. XVI, 318, 424-428.

30. Kantomaa M.T., Tammelin T.H., Näyhä S., Taanila A.M. (2007). Adolescents' physical activity in relation to family income and parents' education. Preventive Medicine 44, 410415.

31. Federico B., Falese L., Capelli G. (2009). Socio-economic inequalities in physical activity practice among Italian children and adolescents: a cross-sectional study. Zeitschrift Für Gesundheitswissenschaften 17(6), 377-384.

32. Lewandowska J., Piechaczek H., Syta A., Łukaszewska L. (2003). Social determinants of the level and form of physical activity and its somatic effects in children from Warsaw. In A. Dąbrowski (ed.), Polish Citizens' Participation in Active Leisure (pp. 340-354). Warszawa: AWF Warszawa, Wyższa Szkoła w Płocku, Wydawnictwo Naukowe Novum. [in Polish]

Submitted:July 24, 2014

Accepted: October 28, 2014 